reversals during the second half of a $10-\mathrm{min}$ trial. Torii (1960) reported a similar decline in reversal rate from an unpublished study of figure-ground reversals. Although wide differences in stimuli and methodology prevent any direct comparison, these results were consistent with those of the present experiment.

More recently, Ammons \& Ammons $(1965,1967)$ found that the reversal rate of the Necker cube decreased during prolonged continuous viewing, following a 5-min rest. However, their Ss were instructed to reverse perspective as rapidly as possible, and this may have been a major factor in their results. No statistical tests were reported by Ammons and Ammons, but their graphs indicated a steady decline in the reversal rate curve over the last $7 \mathrm{~min}$ of an 8 - $\mathrm{min}$ trial.

By comparison with the above studies, the total observation time in the present experiment was quite short $(\bar{X}=3.45 \mathrm{~min})$. However, the use of a rotating stimulus could account for the early decline in reversal rate. Reversals tend to occur much more rapidly in the case of plane figures, and the frequent changes of percept would be expected to hold the attention more readily. In addition, some $S s$ in experiments with the Howard cube have reported a tendency to track visually the path of rotation of the cube. Such tracking responses would reduce the number of reversals recorded by extending the duration of the PI phase, since reversals tend to occur only during periods of steady fixation.

It is suggested that the decline in reversal rate observed in previous studies may result from fluctuations in perspective duration, rather than from any systematic increase in the duration of either phase. Further investigation is needed to clarify the effects of prolonged observation of reversible figures, and to establish the asy mp totic level of the Pl phase.

\section{REFERENCES}

AMMONS, R. B., \& AMMONS, C. H. Learning and performance of rotary pursuit and reversal of perspective with continuous practice and a single rest. Paper presented to Rocky Mountain Psychological Association, 1965.

AMMONS, C. H., \& AMMONS, R. B. Differences in reversible perspective performance related to proficiency. Paper presented to Rocky Mountain Psychological Association, May 1967.

BRUNER, J., POSTMAN, L., \& MOSTELLLR,F. $A$ note on the measurement of reversals of perspective. Psychometrika, 1950, 15,63-72.

HOWARD, I. P. An investigation of a satiation process in the reversible perspective of revolving skeletal shapes. Quarterly Journal of Experimental Psychology, 1961, 13, 19-33.

PRICE, J. R. Perspective duration of a plane reversible figure. Psychonomic Science, 1967a, 9,623-624.

PRICE, J.R. Two components of reversal rate for a rotating skeletal cubc: "Conditioned satiation." Australian Joumal of Psychology, 1967b, 19, $261-270$.

TORII, S. Figure-ground reversals under succes-

\title{
The effects of perceptual organization on performance in classification tasks
}

\section{HAROLD D. FISHBEIN and PAUL FINGERMAN, University of Cincinnati, Cincinnati, Ohio 45221}

Three experiments are reported which investigated the relationship between stimulus organization and performance in classification tasks. In Experiment 1, a conjunctive solution involving proximate stimuli was learned more rapidly than a solution involving nonproximate stimuli. In Experiments 2 and 3, type of solution (similar stimuli vs nonsimilar stimuli in Experiment 2; and unique vs nonunique stimuli in Experiment 3) and number of dimensions had interaction effects on rate of learning.

The major purpose of this paper was to indicate ways in which to study the relationship between the perceptual organization of the to-be-categorized stimulus material and performance in classification (concept identification) tasks. Hence, the experiments reported should be viewed more as demonstrations of a technique than as final answers to the issues which were raised. These concerns stemmed from previous findings (e.g., Bourne, 1966; Shepard, Hovland, \& Jenkins, 1961), which showed that perceptual factors influenced classification performance. In the Shepard et al study, for example, Ss learned a conjunctive concept significantly faster when the stimulus dimensions were imbedded in a single figure than when they were distributed across three figures.

Three experiments are reported here. The first deals with the Gestalt grouping principle of proximity (Kohler, 1947). This principle states that stimuli which are in close proximity to each other are seen as a group. It was hypothesized that a conjunctive solution which involved proximate stimuli would be attained faster than a conjunctive solution which involved adjacent nonproximate stimuli. The second experiment deals with the Gestalt grouping principle of similarity (Kohler, 1947). This principle states that in a stimulus array, similar stimuli are seen as a group. It was hypothesized that a conjunctive solution which involved similar stimuli would be attained faster than a conjunctive solution which involved nonsimilar stimuli. The third experiment deals with the von Restorf effect (Kohler, 1947): In an otherwise homogeneous stimulus array, unique stimuli are perceived as a group. It was hypothesized that a conjunctive solution which involved unique stimuli would be attained faster than a conjunctive solution which involved nonunique stimuli. The overriding view of the three experiments is that perceptual organization is probably important in determining either the order in which Ss test alternative solutions and/or in determining which information will be remembered.

\section{EXPERIMENT}

Subjects and Procedure

This experiment investigated the effects of the Gestalt grouping principle of proximity. Ss were 120 undergraduate students enrolled in introductory psychology courses at the University of Cincinnati, run in a 2 by 3 factorial design experiment.

Four decks of $203 \times 5$ cards were constructed containing either threedimension, two-value stimuli, fourdimension, two-value stimuli, sixdimension, two-value stimuli, or eightdimension, two-value stimuli. A dimension was defined as the fixed location of a letter on the stimulus card. Each location was occupied by either an " $A$ " or "B"-the two values of each dimension. The three, four, six, or eight locations were strung out on a line parallel to the long sides of the card. This type of stimulus display is similar to that used by Glanzer, Huttenlocker, \& Clark (1963).

The locations of the letters on the four-, six-, and eight-dimension cards were juxtaposed so that, introspectively, the lettcrs appeared as pairs. This was accomplished by using a single typewriter space (pica) between the first and second, third and fourth, etc., location, and three spaces between the second and third, fourth and fifth, etc., locations, e.g., 
A B A A B A B B. The locations of the letters on the three-dimension cards were evenly spaced.

The Ss were run on two practice tasks with the three-dimension deck of cards, and one test task with one of the remaining decks, all under a conjunctive rule. The conjunction involved two adjacent letter locations, e.g., first and second, second and third, etc. All test tasks had a solution of "BB", i.e., cards with BB in the critical locations were classified as "ones," and cards with any other letter combinations in those locations were classified as "twos."

Two types of solutions were employed, paired and unpaired. An unpaired solution was one in which the solution involved adjacent locations separated by three typewriter spaces, i.e., letters which appeared unpaired, and a paired solution was one in which the solutions involved adjacent locations separated by one typewriter space, i.e., letters which appeared paired.

The Ss were run under self-paced conditions. The E continued to present one card at a time until $\mathrm{S}$ reached criterion, i.e., correctly classified 12 consecutive cards, or until $S$ had been presented 150 cards. The Ss who failed to reach criterion were assigned a score of 150 . The score of those who reached criterion was the trial number of the last error.

\section{Results}

The major results were that, overall, Ss took fewer trials to reach criterion under the paired than unpaired solutions, and that within a type of solution, the fourdimension tasks were easier than the six-and eight-dimension tasks. The mean trials to criterion were: $12.4,43.5$, and 40.2 for the four-, six-, and eight-dimension paired solutions, respectively; and 58.0, 111.9, and 112.2 for the four-, six-, and eightdimension unpaired solutions, respectively. An analysis of variance confirms the above observations: $F(2,114)=9.28, p<.01$ for dimension effects; $F(1,114)=46.12$, $\mathrm{p}<.01$ for type of solution effects; and $\mathrm{F}(2,114)<1.0, \mathrm{p}>.20$ for the interaction. EXPERIMENT 2

\section{Subjects and Procedure}

This experiment investigated the effects of the Gestalt grouping principle of similarity. The number of Ss, the type of stimulus materials, the type of solution, i.e., conjunction between two adjacent locations, and the experimental procedures were identical with those of Experiment 1. The primary difference was in the construction of the decks of cards. In this experiment, the locations of the positions were evenly spaced. The test task had a solution of either "AA" in the middle two locations (the "similar" solution) or "AB" in the middle two locations (the "nonsimi- lar" solution), e.g., in the four-dimension task, the second and third locations were the basis for solution.

\section{Results}

The major results were that, overall, Ss took fewer trials to reach criterion under the similar than under the nonsimilar solutions, that within a given type of solution, the four-dimension tasks were easier than the six- and eight-dimension tasks, and that there was an interaction between the type of solution and the number of dimensions, such that similar solutions were easier than nonsimilar solutions with four and eight dimensions and were more difficult with six dimensions. The mean trials to criterion were: $19.5,74.75$, and 64.15 for the four-, six-, and eight-dimension similar solutions, respectively; and $51.35,48.60$, and 90.35 for the four-, six-, and eight-dimension nonsimilar solutions, respectively. An analysis of variance tended to confirm the above observations: $F(1,114)=1.39, p>.05$ for type of solution effects; $F(2,114)=7.25$, $\mathrm{p}<.01$ for the dimension effects; and $F(2,114)=4.14, p<.05$ for the interaction.

\section{EXPERIMENT 3}

Subjects and Procedure

This experiment investigated the effects of stimulus novelty (the von Restorf effect). Ss were 96 undergraduate students. The type of solution, i.e., use of the conjunctive rule, and the experimental procedure were identical with those of Experiments 1 and 2. The primary differences were in the construction of the cards and in the number of different letters (values) used. Four-, six-, and eightdimension, four-value cards were constructed according to the following scheme: Each deck in Experiment 2 was used to generate two decks for this experiment. For the first deck, the letter " $X$ " was submitted wherever an " $A$ " appeared in either of the first two locations, and the letter "Y" was substituted wherever a " $B$ " appeared in either of the first two locations. For the second deck, the same thing was done with the last two locations. The locations were evenly spaced. The test task had a solution of either " $X Y$ " in the first two or last two locations (the "unique" solution), or "AB" in the first two or last two locations (the "nonunique" solution). It should be noted that with four-dimension tasks, neither "XY" nor "AB" is unique. Hence, the four-dimension task can be viewed as a control for letter saliency. Each $S$ received two three-dimension, two-value sample tasks, one utilizing As and Bs, and the other using $\mathrm{Xs}$ and $\mathrm{Ys}$.

\section{Results}

The major results were that, overall (pooling across location of solution), Ss took fewer trials to reach criterion under the "XY" than under the " $A B$ " solution, and there was an interaction between type of solution and number of dimensions on performance. The mean trials to criterion were: $32.8,62.1$, and 14.1 for the four-, six-, and eight-dimension "XY" solutions, respectively; and $62.6,40.9$, and 56.9 for the four-, six-, and eight-dimension "AB" solutions, respectively. An analysis of variance tended to confirm the above observations: $F(2,90)=1.28, p>.20$ for the dimension effects; $F(1,90)=2.73$, $p>.10$ for type of solution (XY vs $A B$ ) effects; and $F(2,90)=3.13, p<.05$ for the interaction.

\section{DISCUSSION}

From Experiment 1, it is clear that the solution involving proximate locations was easier than the solution involving nonproximate locations, confirming the experimental hypothesis. This finding is consistent with the Shepard et al results, and suggests an explanation for them. That is, in the Shepard et al experiment, the distributed dimensions stimuli produced less salient solutions than the combined dimensions stimuli.

In both Experiments 2 and 3, perceptual organization and number of dimensions had interaction effects, which failed to confirm the experimental hypotheses. Moreover, in Experiment 3, where neither $X Y$ nor $A B$ were unique stimuli (the four-dimension task), $X Y$ was an easier solution. This makes interpretation of that experiment even more difficult. At least two possible explanations of these findings exist. First, that the experimental manipulations in Experiments 2 and 3 were ineffective and that the results can be attributed to chance. The dramatic performance difference between $X Y$ and $A B$ solutions on the eight-dimension task in Experiment 3 argues against this notion. The second possibility is that the experimental manipulations were effective an that the findings are relaible. This implic that sometimes perceptual grouping he intraction effects with task complexit (number of dimensions) and sometimes $\mathrm{i}$ does not (Experiment 1). A parsimoniou explanation of these differences is no obvious.

\section{REFERENCES}

BOURNE, L., JR. Human conceptual behavior. Boston: Allyn \& Bacon, 1966.

GLANZER, M., HUTTENLOCKER, J., \& CLARK, W. H. Systematic operations in solving concept problems. Psychological Monographs, 1963, 77 (1, Whole No. 564).

KOHLER, W. Gestalt psychology. New York: Liveright, 1947.

SHEPARD, R. N., HOVLAND, C. L., \& JENKINS, M. J. Learning and memorization of classification. Psychological Monographs, 1961, 75 (13, Whole No. 517). 